\title{
THE OPTIMAL PERTURBATION BOUNDS FOR THE WEIGHTED MOORE-PENROSE INVERSE*
}

\author{
WEI-WEI XU $\mathrm{XU}^{\dagger}$, LI-XIA CAI ${ }^{\ddagger}$, AND WEN LI ${ }^{\S}$
}

\begin{abstract}
In this paper, we obtain optimal perturbation bounds of the weighted Moore-Penrose inverse under the weighted unitary invariant norm, the weighted $Q$-norm and the weighted $F$-norm, and thereby extend some recent results.
\end{abstract}

Key words. Weighted Moore-Penrose inverse, Weighted unitary invariant norm, Weighted $Q$-norm, Weighted $F$-norm.

AMS subject classifications. 15A09, 15A18, 15A24.

1. Introduction. Let $\mathbb{C}^{m \times n}$ be the set of complex $m \times n$ matrices and $\mathbb{C}_{r}^{m \times n}$ be the subset consisting of all matrices in $\mathbb{C}^{m \times n}$ of rank $r$. Let $A \in \mathbb{C}^{m \times n}$. We denote $\|A\|,\|A\|_{2},\|A\|_{Q}$ and $\|A\|_{F}$ by the unitary invariant norm, spectral norm, $Q$-norm and $F$-norm of $A$, respectively. The conjugate transformation and the Moore-Penrose generalized inverse of a matrix $A$ are denoted by $A^{*}$ and $A^{\dagger}$, respectively.

Weighted problems, such as the weighted generalized inverse problem and the weighted least squares problem, draw more and more attention, see e.g., [2, 4, 8, 12]. A generalization of the generalized inverse is the weighted Moore-Penrose inverse of an arbitrary matrix which has many applications in numerical computation, statistics, prediction theory, control systems and analysis and curve fitting, see e.g., [1, 9, 14]. There have been many numerical methods for the computation of the weighted MoorePenrose inverse, see e.g., $[6,7,10,11]$. It is an interesting problem to determine how the weighted Moore-Penrose inverse is transformed under perturbation. Answers to this problem will have application in numerical computation, prediction theory and curve fitting. Therefore, it is of significance to estimate the optimal perturbation bounds of the weighted Moore-Penrose inverse. The weighted unitary invariant norm

\footnotetext{
* Received by the editors on October 17, 2010. Accepted for publication on May 14, 2011. Handling Editor: Miroslav Fiedler.

$\dagger$ Institute of Computational Mathematics and Scientific/Engineering Computing, Academy of Mathematics and Systems Science, Chinese Academy of Sciences, PO Box 2719, Beijing 100190, P.R. China (xww@lsec.cc.ac.cn).

${ }^{\ddagger}$ School of Mathematical Sciences, South China Normal University, Guangzhou, 510631 China (clx041@163.com).

${ }^{\S}$ School of Mathematical Sciences, South China Normal University, Guangzhou, 510631 China (liwen@scnu.edu.cn). The work was supported in part by Research Fund for the Doctoral Program of Higher Education of China (Grant No. 20104407110001).
} 
is a more general norm and in terms of this norm, the bounds for the weighted MoorePenrose inverse can be characterized by weighted singular values $((M, N)$ singular values). Recently, much effort has been made for estimating perturbation bounds of the Moore-Penrose inverse, see e.g., [5, 9, 13, 14]. In [13], Wedin presented the perturbation bounds of the Moore-Penrose inverse under a general unitarily invariant norm, the spectral norm and Frobenius norm, respectively. Meng and Zheng in [5] obtained the optimal perturbation bounds for the Moore-Penrose inverse under the Frobenius norm. Cai et al. in [3] obtained the additive and multiplicative perturbation bounds for the Moore-Penrose inverse under the unitary invariant norm and the $Q$ norm, which improves the corresponding results in [13]. In this paper, we will focus our attention on optimal perturbation bounds for the weighted Moore-Penrose inverse in the weighted unitary invariant norm, the weighted $Q$-norm and the weighted $F$ norm and thereby extend the corresponding results in [3] and [5].

We first introduce some basic definitions:

Definition 1.1. [3] A unitary invariant norm $\|\cdot\|$ is called a $Q$-norm if there exists another unitarily invariant norm $\|\cdot\|^{\prime}$ such that $\|Y\|=\left(\left\|Y^{*} Y\right\|^{\prime}\right)^{\frac{1}{2}}$, which is denoted by $\|\cdot\|_{Q}$.

Note that $F$-norm and 2-norm are $Q$-norms.

Definition 1.2. [15] For an arbitrary matrix $A \in \mathbb{C}^{m \times n}$, there is a unique matrix $X \in \mathbb{C}^{n \times m}$ satisfying the following equalities:

- $A X A=A$;

- $X A X=X$;

- $(M A X)^{*}=M A X$;

- $(N X A)^{*}=N X A$.

Then matrix $X$ is called a weighted Moore-Penrose inverse of $A$ and denoted by $X=A_{M N}^{\dagger}$. Here $M$ and $N$ are the given Hermitian positive definite matrices, which are called weighted matrices.

Definition 1.3. [15] Let $A \in \mathbb{C}^{m \times n}$. Then the following norms

- $\|A\|_{(M N)}=\left\|M^{\frac{1}{2}} A N^{-\frac{1}{2}}\right\|$;

- $\|A\|_{F(M N)}=\left\|M^{\frac{1}{2}} A N^{-\frac{1}{2}}\right\|_{F}$;

- $\|A\|_{Q(M N)}=\left\|M^{\frac{1}{2}} A N^{-\frac{1}{2}}\right\|_{Q}$;

- $\|A\|_{M N}=\left\|M^{\frac{1}{2}} A N^{-\frac{1}{2}}\right\|_{2}$,

are called the weighted unitary invariant norm, the weighted $F$-norm, the weighted $Q$-norm and the weighted spectral norm of $A$, respectively.

Definition 1.4. [15] Let $A \in \mathbb{C}_{r}^{m \times n}$. The $(M, N)$ weighted singular value de- 
composition (MN-SVD) of $A \in \mathbb{C}_{r}^{m \times n}$ is expressed as follows:

$$
A=U\left(\begin{array}{cc}
\Sigma & \mathbf{0} \\
\mathbf{0} & \mathbf{0}
\end{array}\right) V^{*}=U_{1} \Sigma V_{1}^{*},
$$

where $U=\left(U_{1}, U_{2}\right) \in \mathbb{C}^{m \times n}$ and $V=\left(V_{1}, V_{2}\right) \in \mathbb{C}^{n \times n}$ satisfy $U^{*} M U=I_{m}$ and $V^{*} N^{-1} V=I_{n}, \Sigma=\operatorname{diag}\left(\sigma_{1}, \ldots, \sigma_{r}\right), \sigma_{i}=\sqrt{\lambda_{i}}$ and $\lambda_{1} \geq \cdots \geq \lambda_{r}>0$ are the nonzero eigenvalues of $N^{-1} A^{*} M A$. Then $\sigma_{1}, \ldots, \sigma_{r}>0$ are called the nonzero $(M, N)$ weighted singular values of $A$.

The rest of this paper is organized as follows. In Section 2, we give some lemmas, which are useful to deduce our main results. In Sections 3 and 4, we consider the additive and multiplicative perturbation of the weighted Moore-Penrose inverse. Some new bounds for additive and multiplicative perturbation under the norms $\|\cdot\|_{(M N)}$, $\|\cdot\|_{Q(M N)}$ and $\|\cdot\|_{F(M N)}$ are presented, which extends the corresponding ones in [5] and [13]. In Section 5, we give some numerical examples to illustrate the optimality of our given bounds under the weighted $Q$-norm and $F$-norm, respectively. Finally, in Section 6 we give concluding remarks.

2. Preliminaries. In this section we give some lemmas, which are useful to deduce our main results.

Lemma 2.1. [12] Let A have $M N-S V D$ (1.1). Then

(1) $A_{M N}^{\dagger}=N^{-1} V_{1} \Sigma^{-1} U_{1}^{*} M$;

(2) $\left\|A_{M N}^{\dagger}\right\|_{N M}=\frac{1}{\sigma_{r}}$.

LEMma 2.2. [3] Let $B$ have the block form

$$
B=\left(\begin{array}{ll}
B_{11} & B_{12} \\
B_{21} & B_{22}
\end{array}\right) .
$$

Then

$$
\|B\|_{Q}^{2} \leq\left\|B_{11}\right\|_{Q}^{2}+\left\|B_{12}\right\|_{Q}^{2}+\left\|B_{21}\right\|_{Q}^{2}+\left\|B_{22}\right\|_{Q}^{2} .
$$

Lemma 2.3. [3] Let $B_{1}$ and $B_{2}$ be two Hermitian matrices and let $P$ be a complex matrix. Suppose that there are two disjoint intervals separated by a gap of width at least $\eta$, where one interval contains the spectrum of $B_{1}$ and the other contains that of $B_{2}$. If $\eta>0$, then there exists a unique solution $X$ to the matrix equation $B_{1} X-X B_{2}=P$ and moreover,

$$
\|X\| \leq \frac{1}{\eta}\|P\|
$$


Lemma 2.4. [3] Let $W \in \mathbb{C}^{n \times n}$ be a unitary matrix with the block form

$$
W=\left(\begin{array}{ll}
W_{11} & W_{12} \\
W_{21} & W_{22}
\end{array}\right)
$$

where $W_{11} \in \mathbb{C}^{r \times r}, W_{22} \in \mathbb{C}^{(n-r) \times(n-r)}, 1 \leq r<n$. Then $\left\|W_{12}\right\|=\left\|W_{21}\right\|$ for any unitarily invariant norm.

3. Additive perturbation bounds. In this section, we will present optimal additive perturbation bounds of the weighted Moore-Penrose inverse under the weighted unitarily invariant norm, the weighted $Q$-norm and the weighted $F$-norm, respectively.

Theorem 3.1. Let $A \in \mathbb{C}_{r}^{m \times n}$ and $B=A+E \in \mathbb{C}_{s}^{m \times n}$. Then

$$
\begin{aligned}
\left\|B_{M N}^{\dagger}-A_{M N}^{\dagger}\right\|_{(N M)} \leq & \left(\left\|A_{M N}^{\dagger}\right\|_{N M}\left\|B_{M N}^{\dagger}\right\|_{N M}+\max \left\{\left\|A_{M N}^{\dagger}\right\|_{N M}^{2},\right.\right. \\
& \left.\left.\left\|B_{M N}^{\dagger}\right\|_{N M}^{2}\right\}\right)\|E\|_{(M N)} .
\end{aligned}
$$

Proof. Let $A$ and $B$ have the following $(M, N)$ weighted singular value decompositions:

$$
A=U\left(\begin{array}{cc}
\Sigma_{1} & \mathbf{0} \\
\mathbf{0} & \mathbf{0}
\end{array}\right) V^{*}=U_{1} \Sigma_{1} V_{1}^{*}, B=\tilde{U}\left(\begin{array}{cc}
\tilde{\Sigma}_{1} & \mathbf{0} \\
\mathbf{0} & \mathbf{0}
\end{array}\right) \tilde{V}^{*}=\tilde{U}_{1} \tilde{\Sigma}_{1} \tilde{V}_{1}^{*},
$$

where $U=\left(U_{1}, U_{2}\right), \tilde{U}=\left(\tilde{U}_{1}, \tilde{U}_{2}\right) \in \mathbb{C}^{m \times m}, V=\left(V_{1}, V_{2}\right), \tilde{V}=\left(\tilde{V}_{1}, \tilde{V}_{2}\right) \in$ $\mathbb{C}^{n \times n}$ satisfy $U^{*} M U=I_{m}, \tilde{U}^{*} M \tilde{U}=I_{m}, V^{*} N^{-1} V=I_{n}$ and $\tilde{V}^{*} N^{-1} \tilde{V}=I_{n}$, $\Sigma_{1}=\operatorname{diag}\left(\sigma_{1}, \ldots, \sigma_{r}\right), \quad \tilde{\Sigma}_{1}=\operatorname{diag}\left(\tilde{\sigma}_{1}, \ldots, \tilde{\sigma}_{s}\right)$ with $\sigma_{1} \geq \cdots \geq \sigma_{r}>0$ and $\tilde{\sigma}_{1} \geq \cdots \geq$ $\tilde{\sigma}_{s}>0$.

By (3.2) we have

$$
E=B-A=\tilde{U}_{1} \tilde{\Sigma}_{1} \tilde{V}_{1}^{*}-U_{1} \Sigma_{1} V_{1}^{*}
$$

By the MN-SVDs (3.2) of $A$ and $B$ we know that $M^{\frac{1}{2}} U, M^{\frac{1}{2}} \tilde{U}, N^{-\frac{1}{2}} V$ and $N^{-\frac{1}{2}} \tilde{V}$ are unitary matrices. Hence from (3.3) one may deduce that

$$
\begin{aligned}
\tilde{\Sigma}_{1} \tilde{V}_{1}^{*} N^{-1} V_{1}-\tilde{U}_{1}^{*} M U_{1} \Sigma_{1} & =\tilde{U}_{1}^{*} M E N^{-1} V_{1}, \\
U_{2}^{*} M \tilde{U}_{1} \tilde{\Sigma}_{1} & =U_{2}^{*} M E N^{-1} \tilde{V}_{1}, \\
\Sigma_{1} V_{1}^{*} N^{-1} \tilde{V}_{2} & =-U_{1}^{*} M E N^{-1} \tilde{V}_{2} .
\end{aligned}
$$

It follows from (3.4) that

$$
\tilde{V}_{1}^{*} N^{-1} V_{1} \Sigma_{1}^{-1}-\tilde{\Sigma}_{1}^{-1} \tilde{U}_{1}^{*} M U_{1}=\tilde{\Sigma}_{1}^{-1} \tilde{U}_{1}^{*} M E N^{-1} V_{1} \Sigma_{1}^{-1} .
$$


By Lemma 2.1 we obtain $A_{M N}^{\dagger}=N^{-1} V_{1} \Sigma_{1}^{-1} U_{1}^{*} M$ and $B_{M N}^{\dagger}=N^{-1} \tilde{V}_{1} \tilde{\Sigma}_{1}^{-1} \tilde{U}_{1}^{*} M$. Then, by Definition 1.3, we have

$$
\begin{aligned}
\left\|B_{M N}^{\dagger}-A_{M N}^{\dagger}\right\|_{(N M)} & =\left\|N^{\frac{1}{2}}\left(N^{-1} \tilde{V}_{1} \tilde{\Sigma}_{1}^{-1} \tilde{U}_{1}^{*} M-N^{-1} V_{1} \Sigma_{1}^{-1} U_{1}^{*} M\right) M^{-\frac{1}{2}}\right\| \\
& =\left\|\tilde{V}^{*} N^{-\frac{1}{2}}\left(N^{-\frac{1}{2}} \tilde{V}_{1} \tilde{\Sigma}_{1}^{-1} \tilde{U}_{1}^{*} M^{\frac{1}{2}}-N^{-\frac{1}{2}} V_{1} \Sigma_{1}^{-1} U_{1}^{*} M^{\frac{1}{2}}\right) M^{\frac{1}{2}} U\right\| \\
& =\left\|\tilde{V}^{*}\left(N^{-1} \tilde{V}_{1} \tilde{\Sigma}_{1}^{-1} \tilde{U}_{1}^{*} M-N^{-1} V_{1} \Sigma_{1}^{-1} U_{1}^{*} M\right) U\right\| \\
& =\left\|\left(\begin{array}{c}
\tilde{V}_{1}^{*} \\
\tilde{V}_{2}^{*}
\end{array}\right)\left(N^{-1} \tilde{V}_{1} \tilde{\Sigma}_{1}^{-1} \tilde{U}_{1}^{*} M-N^{-1} V_{1} \Sigma_{1}^{-1} U_{1}^{*} M\right)\left(U_{1}, U_{2}\right)\right\| \\
& =\left\|\left(\begin{array}{cc}
\tilde{\Sigma}_{1}^{-1} \tilde{U}_{1}^{*} M U_{1}-\tilde{V}_{1}^{*} N^{-1} V_{1} \Sigma_{1}^{-1} & \tilde{\Sigma}_{1}^{-1} \tilde{U}_{1}^{*} M U_{2} \\
-\tilde{V}_{2}^{*} N^{-1} V_{1} \Sigma_{1}^{-1} & \mathbf{0}
\end{array}\right)\right\|,
\end{aligned}
$$

from which one may deduce that

$$
\begin{aligned}
\left\|B_{M N}^{\dagger}-A_{M N}^{\dagger}\right\|_{(N M)} & \leq\left\|\left(\begin{array}{cc}
\tilde{\Sigma}_{1}^{-1} \tilde{U}_{1}^{*} M U_{1}-\tilde{V}_{1}^{*} N^{-1} V_{1} \Sigma_{1}^{-1} & \mathbf{0} \\
\mathbf{0} & \mathbf{0}
\end{array}\right)\right\| \\
& +\left\|\left(\begin{array}{cc}
\mathbf{0} & \tilde{\Sigma}_{1}^{-1} \tilde{U}_{1}^{*} M U_{2} \\
-\tilde{V}_{2}^{*} N^{-1} V_{1} \Sigma_{1}^{-1} & \mathbf{0}
\end{array}\right)\right\| .
\end{aligned}
$$

By (3.7) we have

$$
\left\|\left(\begin{array}{cc}
\tilde{\Sigma}_{1}^{-1} \tilde{U}_{1}^{*} M U_{1}-\tilde{V}_{1}^{*} N^{-1} V_{1} \Sigma_{1}^{-1} & \mathbf{0} \\
\mathbf{0} & \mathbf{0}
\end{array}\right)\right\| \leq \frac{1}{\sigma_{r} \tilde{\sigma}_{s}}\left\|M^{\frac{1}{2}} E N^{-\frac{1}{2}}\right\| .
$$

By (3.5) and (3.6) we have

$$
\begin{aligned}
\tilde{\Sigma}_{1}^{-1} \tilde{U}_{1}^{*} M U_{2} & =\tilde{\Sigma}_{1}^{-2}\left(U_{2}^{*} M E N^{-1} \tilde{V}_{1}\right)^{*}, \\
\tilde{V}_{2}^{*} N^{-1} V_{1} \Sigma_{1}^{-1} & =\left(U_{1}^{*} M E N^{-1} \tilde{V}_{2}\right)^{*} \Sigma_{1}^{-2} .
\end{aligned}
$$

Thus

$$
\begin{aligned}
& \left\|\left(\begin{array}{cc}
\mathbf{0} & \tilde{\Sigma}_{1}^{-1} \tilde{U}_{1}^{*} M U_{2} \\
-\tilde{V}_{2}^{*} N^{-1} V_{1} \Sigma_{1}^{-1} & \mathbf{0}
\end{array}\right)\right\| \\
& \quad \leq \max \left\{\frac{1}{\sigma_{r}^{2}}, \frac{1}{\tilde{\sigma}_{s}^{2}}\right\}\left\|\left(\begin{array}{cc}
\mathbf{0} & \left(U_{2}^{*} M E N^{-1} \tilde{V}_{1}\right)^{*} \\
-\left(U_{1}^{*} M E N^{-1} \tilde{V}_{2}\right)^{*} & \mathbf{0}
\end{array}\right)\right\| .
\end{aligned}
$$

Notice that

$$
\begin{aligned}
\left\|\left(\begin{array}{cc}
\mathbf{0} & \left(U_{2}^{*} M E N^{-1} \tilde{V}_{1}\right)^{*} \\
\left(U_{1}^{*} M E N^{-1} \tilde{V}_{2}\right)^{*} & \mathbf{0}
\end{array}\right)\right\| & \leq\left\|\left(\begin{array}{c}
\tilde{V}_{1}^{*} \\
\tilde{V}_{2}^{*}
\end{array}\right) N^{-1} E^{*} M\left(U_{1}, U_{2}\right)\right\| \\
& =\left\|\tilde{V}^{*} N^{-1} E^{*} M U\right\| .
\end{aligned}
$$


Since $M^{\frac{1}{2}} U$ and $N^{-\frac{1}{2}} \tilde{V}$ are unitary matrices, it follows from (3.11) and (3.12) that

$$
\begin{aligned}
\left\|\left(\begin{array}{cc}
\mathbf{0} & \tilde{\Sigma}_{1}^{-1} \tilde{U}_{1}^{*} M U_{2} \\
-\tilde{V}_{2}^{*} N^{-1} V_{1} \Sigma_{1}^{-1} & \mathbf{0}
\end{array}\right)\right\| & \leq \max \left\{\frac{1}{\sigma_{r}^{2}}, \frac{1}{\tilde{\sigma}_{s}^{2}}\right\}\left\|N^{-\frac{1}{2}} E^{*} M^{\frac{1}{2}}\right\| \\
& =\max \left\{\frac{1}{\sigma_{r}^{2}}, \frac{1}{\tilde{\sigma}_{s}^{2}}\right\}\left\|M^{\frac{1}{2}} E N^{-\frac{1}{2}}\right\| \\
& =\max \left\{\frac{1}{\sigma_{r}^{2}}, \frac{1}{\tilde{\sigma}_{s}^{2}}\right\}\|E\|_{(M N)},
\end{aligned}
$$

which together with (3.9), (3.10) and Lemma 2.1(2) deduces (3.1).

Remark 3.2. If we take $M=N=I$, then Theorem 3.1 reduces to

$$
\left\|B^{\dagger}-A^{\dagger}\right\| \leq\left(\left\|A^{\dagger}\right\|_{2}\left\|B^{\dagger}\right\|_{2}+\max \left\{\left\|A^{\dagger}\right\|_{2}^{2},\left\|B^{\dagger}\right\|_{2}^{2}\right\}\right)\|E\|,
$$

which is the result of Theorem 3.1 in [3].

For the $Q_{(N M)}$-norm we provide the following bound.

Theorem 3.3. Let $A \in \mathbb{C}_{r}^{m \times n}$ and $B=A+E \in \mathbb{C}_{s}^{m \times n}$. Then

$$
\begin{aligned}
& \left\|B_{M N}^{\dagger}-A_{M N}^{\dagger}\right\|_{Q(N M)} \\
& \quad \leq \sqrt{\left\|A_{M N}^{\dagger}\right\|_{N M}^{4}+\left\|B_{M N}^{\dagger}\right\|_{N M}^{4}+\left\|A_{M N}^{\dagger}\right\|_{N M}^{2}\left\|B_{M N}^{\dagger}\right\|_{N M}^{2}}\|E\|_{Q(M N)} .
\end{aligned}
$$

Proof. The bound (3.14) follows immediately from Lemma 2.2, (3.4)-(3.6) and (3.8).

Remark 3.4. If we take $M=N=I$, then Theorem 3.3 reduces to

$$
\left\|B^{\dagger}-A^{\dagger}\right\|_{Q} \leq \sqrt{\left\|A^{\dagger}\right\|_{2}^{4}+\left\|B^{\dagger}\right\|_{2}^{4}+\left\|A^{\dagger}\right\|_{2}^{2}\left\|B^{\dagger}\right\|_{2}^{2}}\|E\|_{Q},
$$

which is the result of Theorem 3.2 in [3].

Theorem 3.5. Let $A \in \mathbb{C}_{r}^{m \times n}$ and $B=A+E \in \mathbb{C}_{s}^{m \times n}$. Then

$$
\left\|B_{M N}^{\dagger}-A_{M N}^{\dagger}\right\|_{F(N M)} \leq \max \left\{\left\|A_{M N}^{\dagger}\right\|_{N M}^{2},\left\|B_{M N}^{\dagger}\right\|_{N M}^{2}\right\}\|E\|_{F(M N)} .
$$

Proof. It follows from (3.3) that

$$
\begin{array}{ll}
(3.16) & U_{1}^{*} M \tilde{U}_{1} \tilde{\Sigma}_{1}-\Sigma_{1} V_{1}^{*} N^{-1} \tilde{V}_{1}=U_{1}^{*} M E N^{-1} \tilde{V}_{1}, \\
(3.17) & \tilde{U}_{2}^{*} M U_{1} \Sigma_{1}=-\tilde{U}_{2}^{*} M E N^{-1} V_{1}, U_{2}^{*} M \tilde{U}_{1} \tilde{\Sigma}_{1}=U_{2}^{*} M E N^{-1} \tilde{V}_{1}, \\
(3.18) & \tilde{\Sigma}_{1} \tilde{V}_{1}^{*} N^{-1} V_{2}=\tilde{U}_{1}^{*} M E N^{-1} V_{2}, \Sigma_{1} V_{1}^{*} N^{-1} \tilde{V}_{2}=-U_{1}^{*} M E N^{-1} \tilde{V}_{2} .
\end{array}
$$


By (3.16), we have

$$
\Sigma_{1}^{-1} U_{1}^{*} M \tilde{U}_{1}-V_{1}^{*} N^{-1} \tilde{V}_{1} \tilde{\Sigma}_{1}^{-1}=\Sigma_{1}^{-1} U_{1}^{*} M E N^{-1} \tilde{V}_{1} \tilde{\Sigma}_{1}^{-1} .
$$

It is easy to see that

$$
\begin{aligned}
\left\|B_{M N}^{\dagger}-A_{M N}^{\dagger}\right\|_{F(N M)} & =\left\|N^{\frac{1}{2}}\left(N^{-1} \tilde{V}_{1} \tilde{\Sigma}_{1}^{-1} \tilde{U}_{1}^{*} M-N^{-1} V_{1} \Sigma_{1}^{-1} U_{1}^{*} M\right) M^{-\frac{1}{2}}\right\|_{F} \\
& =\left\|V^{*} N^{-\frac{1}{2}}\left(N^{-\frac{1}{2}} \tilde{V}_{1} \tilde{\Sigma}_{1}^{-1} \tilde{U}_{1}^{*} M^{\frac{1}{2}}-N^{-\frac{1}{2}} V_{1} \Sigma_{1}^{-1} U_{1}^{*} M^{\frac{1}{2}}\right) M^{\frac{1}{2}} \tilde{U}\right\|_{F} \\
& =\left\|V^{*}\left(N^{-1} \tilde{V}_{1} \tilde{\Sigma}_{1}^{-1} \tilde{U}_{1}^{*} M-N^{-1} V_{1} \Sigma_{1}^{-1} U_{1}^{*} M\right) \tilde{U}\right\|_{F} \\
& =\left\|\left(\begin{array}{c}
V_{1}^{*} \\
V_{2}^{*}
\end{array}\right)\left(N^{-1} \tilde{V}_{1} \tilde{\Sigma}_{1}^{-1} \tilde{U}_{1}^{*} M-N^{-1} V_{1} \Sigma_{1}^{-1} U_{1}^{*} M\right)\left(\tilde{U}_{1}, \tilde{U}_{2}\right)\right\|_{F} \\
& =\left\|\left(\begin{array}{cc}
V_{1}^{*} N^{-1} \tilde{V}_{1} \tilde{\Sigma}_{1}^{-1}-\Sigma_{1}^{-1} U_{1}^{*} M \tilde{U}_{1} & -\Sigma_{1}^{-1} U_{1}^{*} M \tilde{U}_{2} \\
V_{2}^{*} N^{-1} \tilde{V}_{1} \tilde{\Sigma}_{1}^{-1} & \mathbf{0}
\end{array}\right)\right\|_{F} .
\end{aligned}
$$

It follows from (3.8) and (3.20) that

$$
\begin{aligned}
2\left\|B_{M N}^{\dagger}-A_{M N}^{\dagger}\right\|_{F(N M)}^{2} & =\left\|\tilde{\Sigma}_{1}^{-1} \tilde{U}_{1}^{*} M U_{1}-\tilde{V}_{1}^{*} N^{-1} V_{1} \Sigma_{1}^{-1}\right\|_{F}^{2}+\left\|\tilde{\Sigma}_{1}^{-1} \tilde{U}_{1}^{*} M U_{2}\right\|_{F}^{2} \\
& +\left\|\tilde{V}_{2}^{*} N^{-1} V_{1} \Sigma_{1}^{-1}\right\|_{F}^{2}+\left\|V_{1}^{*} N^{-1} \tilde{V}_{1} \tilde{\Sigma}_{1}^{-1}-\Sigma_{1}^{-1} U_{1}^{*} M \tilde{U}_{1}\right\|_{F}^{2} \\
& +\left\|\Sigma_{1}^{-1} U_{1}^{*} M \tilde{U}_{2}\right\|_{F}^{2}+\left\|V_{2}^{*} N^{-1} \tilde{V}_{1} \tilde{\Sigma}_{1}^{-1}\right\|_{F}^{2},
\end{aligned}
$$

which together with Lemma 2.2, (3.4)-(3.6) and (3.16)-(3.19) yields

$$
\begin{aligned}
2\left\|B_{M N}^{\dagger}-A_{M N}^{\dagger}\right\|_{F(N M)}^{2} & =\left\|\tilde{\Sigma}_{1}^{-1} \tilde{U}_{1}^{*} M E N^{-1} V_{1} \Sigma_{1}^{-1}\right\|_{F}^{2}+\left\|\tilde{\Sigma}_{1}^{-2} \tilde{V}_{1}^{*} N^{-1} E^{*} M U_{2}\right\|_{F}^{2} \\
& +\left\|\Sigma_{1}^{-1} U_{1}^{*} M E N^{-1} \tilde{V}_{1} \tilde{\Sigma}_{1}^{-1}\right\|_{F}^{2}+\left\|\tilde{V}_{2}^{*} N^{-1} E^{*} M U_{1} \Sigma_{1}^{-2}\right\|_{F}^{2} \\
& +\left\|\Sigma_{1}^{-2} V_{1}^{*} N^{-1} E^{*} M \tilde{U}_{2}\right\|_{F}^{2}+\left\|V_{2}^{*} N^{-1} E^{*} M \tilde{U}_{1} \tilde{\Sigma}_{1}^{-2}\right\|_{F}^{2} \\
& \leq \frac{1}{\sigma_{r}^{2} \tilde{\sigma}_{s}^{2}}\left(\left\|\tilde{U}_{1}^{*} M E N^{-1} V_{1}\right\|_{F}^{2}+\left\|U_{1}^{*} M E N^{-1} \tilde{V}_{1}\right\|_{F}^{2}\right) \\
& +\frac{1}{\sigma_{r}^{4}}\left(\left\|\tilde{V}_{2}^{*} N^{-1} E^{*} M U_{1}\right\|_{F}^{2}+\left\|V_{1}^{*} N^{-1} E^{*} M \tilde{U}_{2}\right\|_{F}^{2}\right) \\
& \leq \max \left\{\frac{1}{\sigma_{r}^{4}}, \frac{1}{\tilde{\sigma}_{s}^{4}}\right\}\left(\left\|\tilde{U}_{1}^{*} M E N^{-1} V_{1}\right\|_{F}^{2}+\left\|U_{1}^{*} M E N^{-1} \tilde{V}_{1}\right\|_{F}^{2}\right) \\
& +\left\|\tilde{V}_{1}^{*} N^{-1} E^{*} M U_{2}\right\|_{F}^{2}+\left\|V_{2}^{*} N^{-1} E^{*} M \tilde{U}_{1}\right\|_{F}^{2} \\
& +\left\|\tilde{V}_{2}^{*} N^{-1} E^{*} M U_{1}\right\|_{F}^{2}+\left\|V_{1}^{*} N^{-1} E^{*} M \tilde{U}_{2}\right\|_{F}^{2} \\
& \leq 2 \max \left\{\frac{1}{\sigma_{r}^{4}}, \frac{1}{\tilde{\sigma}_{s}^{4}}\right\}\left\|M^{\frac{1}{2}} E N^{-\frac{1}{2}}\right\|_{F}^{2} \\
& =2 \max \left\{\left\|A_{M N}^{\dagger}\right\|\left\|_{N M}^{4}\right\| B_{M N}^{\dagger} \|_{N M}^{4}\right\}\|E\|_{F(M N)}^{2} .
\end{aligned}
$$

Therefore,

$$
\left\|B_{M N}^{\dagger}-A_{M N}^{\dagger}\right\|_{F(N M)} \leq \max \left\{\left\|A_{M N}^{\dagger}\right\|_{N M}^{2},\left\|B_{M N}^{\dagger}\right\|_{N M}^{2}\right\}\|E\|_{F(M N)},
$$

which implies (3.15) holds. प 
REMARK 3.6. If we take $M=N=I$, then Theorem 3.5 reduces to

$$
\left\|B^{\dagger}-A^{\dagger}\right\|_{F} \leq \max \left\{\left\|A^{\dagger}\right\|_{2}^{2},\left\|B^{\dagger}\right\|_{2}^{2}\right\}\|E\|_{F}
$$

which is the result of Theorem 2.1 in [5].

Now we consider the case that $\operatorname{rank}(A)=\operatorname{rank}(B)$, i.e., $A, B \in \mathbb{C}_{r}^{m \times n}$.

Theorem 3.7. Let $A, B=A+E \in \mathbb{C}_{r}^{m \times n}$. Then

$$
\begin{aligned}
\left\|B_{M N}^{\dagger}-A_{M N}^{\dagger}\right\|_{(N M)} \leq & {\left[\left\|A_{M N}^{\dagger}\right\|_{N M}\left\|B_{M N}^{\dagger}\right\|_{N M}+\left(\left\|A_{M N}^{\dagger}\right\|_{N M}+\left\|B_{M N}^{\dagger}\right\|_{N M}\right)\right.} \\
& \left.\min \left\{\left\|A_{M N}^{\dagger}\right\|_{N M},\left\|B_{M N}^{\dagger}\right\|_{N M}\right\}\right]\|E\|_{(M N)} .
\end{aligned}
$$

Proof. Let

$$
\begin{gathered}
D_{1}=\left(\begin{array}{cc}
\Sigma_{1} & \mathbf{0} \\
\mathbf{0} & \sigma_{r} I
\end{array}\right), D_{2}=\left(\begin{array}{cc}
\tilde{\Sigma}_{1} & \mathbf{0} \\
\mathbf{0} & \tilde{\sigma}_{r} I
\end{array}\right), \text { and } \\
X=\left(\begin{array}{cc}
\tilde{\Sigma}_{1}^{-1} \tilde{U}_{1}^{*} M U_{1}-\tilde{V}_{1}^{*} N^{-1} V_{1} \Sigma_{1}^{-1} & \tilde{\Sigma}_{1}^{-1} \tilde{U}_{1}^{*} M U_{2} \\
-\tilde{V}_{2}^{*} N^{-1} V_{1} \Sigma_{1}^{-1} & \mathbf{0}
\end{array}\right) .
\end{gathered}
$$

Then

$$
\begin{aligned}
X D_{1}+D_{2} X & =\left(\begin{array}{cc}
\tilde{\Sigma}_{1}^{-1} \tilde{U}_{1}^{*} M U_{1} \Sigma_{1}-\tilde{V}_{1}^{*} N^{-1} V_{1} & \sigma_{r} \tilde{\Sigma}_{1}^{-1} \tilde{U}_{1}^{*} M U_{2} \\
-\tilde{V}_{2}^{*} N^{-1} V_{1} & \mathbf{0}
\end{array}\right) \\
& +\left(\begin{array}{cc}
\tilde{U}_{1}^{*} M U_{1}-\tilde{\Sigma}_{1} \tilde{V}_{1}^{*} N^{-1} V_{1} \Sigma_{1}^{-1} & \tilde{U}_{1}^{*} M U_{2} \\
-\tilde{\sigma}_{r} \tilde{V}_{2}^{*} N^{-1} V_{1} \Sigma_{1}^{-1} & \mathbf{0}
\end{array}\right) .
\end{aligned}
$$

From (3.4) it is easy to see that

$$
\begin{aligned}
& \left\|\tilde{\Sigma}_{1}^{-1} \tilde{U}_{1}^{*} M U_{1} \Sigma_{1}-\tilde{V}_{1}^{*} N^{-1} V_{1}\right\| \leq \frac{1}{\tilde{\sigma}_{r}}\left\|M^{\frac{1}{2}} E N^{-\frac{1}{2}}\right\| \leq \frac{1}{\tilde{\sigma}_{r}}\|E\|_{(M N)}, \\
& \left\|\tilde{U}_{1}^{*} M U_{1}-\tilde{\Sigma}_{1} \tilde{V}_{1}^{*} N^{-1} V_{1} \Sigma_{1}^{-1}\right\| \leq \frac{1}{\sigma_{r}}\left\|M^{\frac{1}{2}} E N^{-\frac{1}{2}}\right\| \leq \frac{1}{\sigma_{r}}\|E\|_{(M N)} .
\end{aligned}
$$

Since $\widetilde{U}^{*} M U$ is unitary, by Lemma 2.4 we have

$$
\left\|\widetilde{U}_{1}^{*} M U_{2}\right\|=\left\|\widetilde{U}_{2}^{*} M U_{1}\right\| .
$$

By (3.3) and (3.5), one may deduce that

$$
\widetilde{U}_{2}^{*} M U_{1}=-\widetilde{U}_{2}^{*} M E N^{-1} V_{1} \Sigma_{1}^{-1}
$$


and

$$
\widetilde{U}_{1}^{*} M U_{2}=\widetilde{\Sigma}_{1}^{-1}\left(U_{2}^{*} M E N^{-1} \widetilde{V}_{1}\right)^{*} .
$$

This in turn implies that

$$
\left\|\widetilde{U}_{2}^{*} M U_{1}\right\| \leq \frac{1}{\sigma_{r}}\|E\|_{M N}
$$

and

$$
\left\|\widetilde{U}_{1}^{*} M U_{2}\right\| \leq \frac{1}{\widetilde{\sigma_{r}}}\|E\|_{M N}
$$

respectively. Then

$$
\left\|\tilde{U}_{1}^{*} M U_{2}\right\| \leq \frac{1}{\max \left\{\sigma_{r}, \tilde{\sigma}_{r}\right\}}\|E\|_{(M N)},
$$

and thus

$$
\left\|\sigma_{r} \tilde{\Sigma}_{1}^{-1} \tilde{U}_{1}^{*} M U_{2}\right\| \leq \frac{\sigma_{r}}{\tilde{\sigma}_{r}}\left\|\tilde{U}_{1}^{*} M U_{2}\right\| \leq \frac{\sigma_{r}}{\tilde{\sigma}_{r} \max \left\{\sigma_{r}, \tilde{\sigma}_{r}\right\}}\|E\|_{(M N)}
$$

By an analogous argument, we have

$$
\left\|\tilde{V}_{2}^{*} N^{-1} V_{1}\right\| \leq \frac{1}{\max \left\{\sigma_{r}, \tilde{\sigma}_{r}\right\}}\|E\|_{(M N)}
$$

and

$$
\left\|\tilde{\sigma}_{r} \tilde{V}_{2}^{*} N^{-1} V_{1} \Sigma_{1}^{-1}\right\| \leq \frac{\tilde{\sigma}_{r}}{\sigma_{r}}\left\|\tilde{V}_{2}^{*} N^{-1} V_{1}\right\| \leq \frac{\tilde{\sigma}_{r}}{\sigma_{r} \max \left\{\sigma_{r}, \tilde{\sigma}_{r}\right\}}\|E\|_{(M N)},
$$

which together with (3.8), (3.22)-(3.24) and Lemma 2.3 give the desired result. $\square$

REMARK 3.8. If we take $M=N=I$, then Theorem 3.7 reduces to (3.25) $\left\|B^{\dagger}-A^{\dagger}\right\| \leq\left[\left\|A^{\dagger}\right\|_{2}\left\|B^{\dagger}\right\|_{2}+\left(\left\|A^{\dagger}\right\|_{2}+\left\|B^{\dagger}\right\|_{2}\right) \min \left\{\left\|A^{\dagger}\right\|_{2},\left\|B^{\dagger}\right\|_{2}\right\}\right]\|E\|$, which is the result of Theorem 3.3 in [3].

For the weighted $F$-norm we have:

Theorem 3.9. Let $A, B=A+E \in \mathbb{C}_{r}^{m \times n}$. Then

$$
\left\|B_{M N}^{\dagger}-A_{M N}^{\dagger}\right\|_{F(N M)} \leq\left\|A_{M N}^{\dagger}\right\|_{N M}\left\|B_{M N}^{\dagger}\right\|_{N M}\|E\|_{F(M N)} .
$$

Proof. Since in (1.1) $U^{*} M \tilde{U}, \tilde{V} N^{-1} V$ and $V^{*} N^{-1} \tilde{V}$ are unitary, by Lemma 2.4 we have

$$
\left\|\tilde{U}_{1}^{*} M U_{2}\right\|_{F}=\left\|\tilde{U}_{2}^{*} M U_{1}\right\|_{F},\left\|U_{2}^{*} M \tilde{U}_{1}\right\|_{F}=\left\|U_{1}^{*} M \tilde{U}_{2}\right\|_{F},
$$




$$
\left\|\tilde{V}_{1}^{*} N^{-1} V_{2}\right\|_{F}=\left\|\tilde{V}_{2}^{*} N^{-1} V_{1}\right\|_{F},\left\|V_{2}^{*} N^{-1} \tilde{V}_{1}\right\|_{F}=\left\|V_{1}^{*} N^{-1} \tilde{V}_{2}\right\|_{F} .
$$

It follows from (3.17), (3.18) and (3.27), (3.28) that

$$
\begin{aligned}
2\left\|B_{M N}^{\dagger}-A_{M N}^{\dagger}\right\|_{F(N M)}^{2} & =\left\|\tilde{\Sigma}_{1}^{-1} \tilde{U}_{1}^{*} M E N^{-1} V_{1} \Sigma_{1}^{-1}\right\|_{F}^{2}+\left\|\Sigma_{1}^{-1} U_{1}^{*} M E N^{-1} \tilde{V}_{1} \tilde{\Sigma}_{1}^{-1}\right\|_{F}^{2} \\
& +\frac{1}{\tilde{\sigma}_{r}^{2}}\left(\left\|\tilde{U}_{1}^{*} M U_{2}\right\|_{F}^{2}+\left\|V_{2}^{*} N^{-1} \tilde{V}_{1}\right\|_{F}^{2}\right) \\
& +\frac{1}{\sigma_{r}^{2}}\left(\left\|\tilde{V}_{2}^{*} N^{-1} V_{1}\right\|_{F}^{2}+\left\|U_{1}^{*} M \tilde{U}_{2}\right\|_{F}^{2}\right) \\
& \leq \frac{1}{\tilde{\sigma}_{r}^{2} \sigma_{r}^{2}}\left(\left\|\tilde{U}_{1}^{*} M E N^{-1} V_{1}\right\|_{F}^{2}+\left\|U_{1}^{*} M E N^{-1} \tilde{V}_{1}\right\|_{F}^{2}\right) \\
& +\frac{1}{\tilde{\sigma}_{r}^{2}}\left(\left\|\tilde{U}_{2}^{*} M U_{1}\right\|_{F}^{2}+\left\|V_{1}^{*} N^{-1} \tilde{V}_{2}\right\|_{F}^{2}\right) \\
& +\frac{1}{\sigma_{r}^{2}}\left(\left\|\tilde{V}_{1}^{*} N^{-1} V_{2}\right\|_{F}^{2}+\left\|U_{2}^{*} M \tilde{U}_{1}\right\|_{F}^{2}\right) \\
& =\frac{1}{\tilde{\sigma}_{r}^{2} \sigma_{r}^{2}}\left(\left\|\tilde{U}_{1}^{*} M E N^{-1} V_{1}\right\|_{F}^{2}+\left\|U_{1}^{*} M E N^{-1} \tilde{V}_{1}\right\|_{F}^{2}\right) \\
& +\frac{1}{\tilde{\sigma}_{r}^{2}}\left(\left\|\tilde{U}_{2}^{*} M E N^{-1} V_{1} \Sigma_{1}^{-1}\right\|_{F}^{2}+\left\|\Sigma_{1}^{-1} U_{1}^{*} M E N^{-1} \tilde{V}_{2}\right\|_{F}^{2}\right) \\
& +\frac{1}{\sigma_{r}^{2}}\left(\left\|\tilde{\Sigma}_{1}^{-1} \tilde{U}_{1}^{*} M E N^{-1} V_{2}\right\|_{F}^{2}+\left\|U_{2}^{*} M E N^{-1} \tilde{V}_{1} \tilde{\Sigma}_{1}^{-1}\right\|_{F}^{2}\right) \\
& \leq \frac{2}{\tilde{\sigma}_{r}^{2} \sigma_{r}^{2}}\left\|M^{\frac{1}{2}} E N^{-\frac{1}{2}}\right\|_{F}^{2}=2\left\|A_{M N}^{\dagger}\right\|_{N M}^{2}\left\|B_{M N}^{\dagger}\right\|_{N M}^{2}\|E\|_{F(M N)}^{2}, \\
& +
\end{aligned}
$$

which implies (3.26) holds. $\square$

REMARK 3.10. Since

$$
\left\|A_{M N}^{\dagger}\right\|_{N M}\left\|B_{M N}^{\dagger}\right\|_{N M} \leq \max \left\{\left\|A_{M N}^{\dagger}\right\|_{N M}^{2},\left\|B_{M N}^{\dagger}\right\|_{N M}^{2}\right\}
$$

the bound is sharper than the one in (3.15).

For the weighted $Q$-norm we have:

Theorem 3.11. Let $A, B=A+E \in \mathbb{C}_{r}^{m \times n}$. Then

$$
\left\|B_{M N}^{\dagger}-A_{M N}^{\dagger}\right\|_{Q(N M)} \leq \sqrt{3}\left\|A_{M N}^{\dagger}\right\|_{N M}\left\|B_{M N}^{\dagger}\right\|_{N M}\|E\|_{Q(M N)} .
$$

Proof. By (3.20) we have

$$
\left\|B_{M N}^{\dagger}-A_{M N}^{\dagger}\right\|_{Q(N M)}=\left\|\left(\begin{array}{cc}
V_{1}^{*} N^{-1} \tilde{V}_{1} \tilde{\Sigma}_{1}^{-1}-\Sigma_{1}^{-1} U_{1}^{*} M \tilde{U}_{1} & -\Sigma_{1}^{-1} U_{1}^{*} M \tilde{U}_{2} \\
V_{2}^{*} N^{-1} \tilde{V}_{1} \tilde{\Sigma}_{1}^{-1} & \mathbf{0}
\end{array}\right)\right\|_{Q .}
$$


It follows from (3.8), (3.27), (3.28) and Lemma 2.2 that

$$
\begin{aligned}
2\left\|B_{M N}^{\dagger}-A_{M N}^{\dagger}\right\|_{Q(N M)}^{2} & \leq\left\|\tilde{\Sigma}_{1}^{-1} \tilde{U}_{1}^{*} M U_{1}-\tilde{V}_{1}^{*} N^{-1} V_{1} \Sigma_{1}^{-1}\right\|_{Q}^{2}+\left\|\tilde{\Sigma}_{1}^{-1} \tilde{U}_{1}^{*} M U_{2}\right\|_{Q}^{2} \\
& +\left\|\tilde{V}_{2}^{*} N^{-1} V_{1} \Sigma_{1}^{-1}\right\|_{Q}^{2}+\left\|V_{1}^{*} N^{-1} \tilde{V}_{1} \tilde{\Sigma}_{1}^{-1}-\Sigma_{1}^{-1} U_{1}^{*} M \tilde{U}_{1}\right\|_{Q}^{2} \\
& +\left\|\Sigma_{1}^{-1} U_{1}^{*} M \tilde{U}_{2}\right\|_{Q}^{2}+\left\|V_{2}^{*} N^{-1} \tilde{V}_{1} \tilde{\Sigma}_{1}^{-1}\right\|_{Q}^{2},
\end{aligned}
$$

which together with (3.4)-(3.7) and (3.16)-(3.19) gives that

$$
\begin{aligned}
2\left\|B_{M N}^{\dagger}-A_{M N}^{\dagger}\right\|_{Q(N M)}^{2} \leq & \left\|\tilde{\Sigma}_{1}^{-1} \tilde{U}_{1}^{*} M E N^{-1} V_{1} \Sigma_{1}^{-1}\right\|_{Q}^{2}+\left\|\Sigma_{1}^{-1} U_{1}^{*} M E N^{-1} \tilde{V}_{1} \tilde{\Sigma}_{1}^{-1}\right\|_{Q}^{2} \\
& +\frac{1}{\tilde{\sigma}_{r}^{2}}\left(\left\|\tilde{U}_{1}^{*} M U_{2}\right\|_{Q}^{2}+\left\|V_{2}^{*} N^{-1} \tilde{V}_{1}\right\|_{Q}^{2}\right) \\
& +\frac{1}{\sigma_{r}^{2}}\left(\left\|\tilde{V}_{2}^{*} N^{-1} V_{1}\right\|_{Q}^{2}+\left\|U_{1}^{*} M \tilde{U}_{2}\right\|_{Q}^{2}\right) \\
& \leq \frac{1}{\sigma_{r}^{2} \tilde{\sigma}_{r}^{2}}\left(\left\|\tilde{U}_{1}^{*} M E N^{-1} V_{1}\right\|_{Q}^{2}+\left\|U_{1}^{*} M E N^{-1} \tilde{V}_{1}\right\|_{Q}^{2}\right) \\
& +\frac{1}{\tilde{\sigma}_{r}^{2}}\left(\left\|\tilde{U}_{2}^{*} M U_{1}\right\|_{Q}^{2}+\left\|V_{1}^{*} N^{-1} \tilde{V}_{2}\right\|_{Q}^{2}\right) \\
& +\frac{1}{\sigma_{r}^{2}}\left(\left\|\tilde{V}_{1}^{*} N^{-1} V_{2}\right\|_{Q}^{2}+\left\|U_{2}^{*} M \tilde{U}_{1}\right\|_{Q}^{2}\right) \\
& =\frac{1}{\sigma_{r}^{2} \tilde{\sigma}_{r}^{2}}\left(\left\|\tilde{U}_{1}^{*} M E N^{-1} V_{1}\right\|_{Q}^{2}+\left\|U_{1}^{*} M E N^{-1} \tilde{V}_{1}\right\|_{Q}^{2}\right) \\
& +\frac{1}{\tilde{\sigma}_{r}^{2}}\left(\left\|\tilde{U}_{2}^{*} M E N^{-1} V_{1} \Sigma_{1}^{-1}\right\|_{Q}^{2}+\left\|\Sigma_{1}^{-1} U_{1}^{*} M E N^{-1} \tilde{V}_{2}\right\|_{Q}^{2}\right) \\
& +\frac{1}{\sigma_{r}^{2}}\left(\left\|\tilde{\Sigma}_{1}^{-1} \tilde{U}_{1}^{*} M E N^{-1} V_{2}\right\|_{Q}^{2}+\left\|U_{2}^{*} M E N^{-1} \tilde{V}_{1} \tilde{\Sigma}_{1}^{-1}\right\|_{Q}^{2}\right) \\
& \leq \frac{1}{\sigma_{r}^{2} \tilde{\sigma}_{r}^{2}}\left(\left\|\tilde{U}_{1}^{*} M E N^{-1} V_{1}\right\|_{Q}^{2}+\left\|\tilde{U}_{2}^{*} M E N^{-1} V_{1}\right\|_{Q}^{2}\right. \\
& +\left\|\tilde{U}_{1}^{*} M E N^{-1} V_{2}\right\|_{Q}^{2}+\left\|U_{1}^{*} M E N^{-1} \tilde{V}_{1}\right\|_{Q}^{2} \\
& \left.+\left\|U_{1}^{*} M E N^{-1} \tilde{V}_{2}\right\|_{Q}^{2}+\left\|U_{2}^{*} M E N^{-1} \tilde{V}_{1}\right\|_{Q}^{2}\right) \\
& \leq 6\left\|A_{M N}^{\dagger}\right\|_{N M}^{2}\left\|B_{M N}^{\dagger}\right\|_{N M}^{2}\|E\|_{Q(M N)}^{2},
\end{aligned}
$$

which implies that the inequality (3.29) holds. $\square$

Remark 3.12. If we take $M=N=I$, then Theorem 3.11 reduces to

$$
\left\|B^{\dagger}-A^{\dagger}\right\|_{Q} \leq \sqrt{3}\left\|A^{\dagger}\right\|_{2}\left\|B^{\dagger}\right\|_{2}\|E\|_{Q},
$$

which is the result of Theorem 3.4 in [3].

It is easy to see that

$$
\sqrt{3}\left\|A_{M N}^{\dagger}\right\|_{N M}\left\|B_{M N}^{\dagger}\right\|_{N M} \leq \sqrt{\left\|A_{M N}^{\dagger}\right\|_{N M}^{4}+\left\|B_{M N}^{\dagger}\right\|_{N M}^{4}+\left\|A_{M N}^{\dagger}\right\|_{N M}^{2}\left\|B_{M N}^{\dagger}\right\|_{N M}^{2}},
$$

which implies that the bound in (3.29) is sharper than the one in (3.14). 
4. Multiplicative perturbation bounds. In this section, we present optimal multiplicative perturbation bounds of the weighted Moore-Penrose inverse. Let $B$ be a multiplicative perturbed matrix of $A$, i.e., $B=D_{1} A D_{2}$, where $D_{1}$ and $D_{2}$ are $m \times m$ and $n \times n$ nonsingular matrices, then $\operatorname{rank}(A)=\operatorname{rank}(B)$.

Theorem 4.1. Let $A \in \mathbb{C}^{m \times n}$ and $B=D_{1}^{*} A D_{2}$, where $D_{1}$ and $D_{2}$ are respectively $m \times m$ and $n \times n$ nonsingular matrices. Then

$$
\left\|B_{M N}^{\dagger}-A_{M N}^{\dagger}\right\|_{(N M)} \leq \max \left\{\left\|A_{M N}^{\dagger}\right\|_{N M},\left\|B_{M N}^{\dagger}\right\|_{N M}\right\} \Psi_{1}\left(D_{1}, D_{2}\right),
$$

where

$\Psi_{1}\left(D_{1}, D_{2}\right)=\left\|I_{m}-D_{1}^{*}\right\|_{(M M)}+\left\|I_{m}-D_{1}^{-*}\right\|_{(M M)}+\left\|I_{n}-D_{2}\right\|_{(N N)}+\left\|I_{n}-D_{2}^{-1}\right\|_{(N N)}$.

Proof. Let $A$ and $B$ have the MN-SVDs (3.2). Clearly we have

$$
\begin{aligned}
B-A & =B\left(I_{n}-D_{2}^{-1}\right)+\left(D_{1}^{*}-I_{m}\right) A \\
& =\left(I_{m}-D_{1}^{-*}\right) B+A\left(D_{2}-I_{n}\right) .
\end{aligned}
$$

It follows from (3.3) and (4.2) that

$$
\begin{aligned}
\tilde{U}_{1} \tilde{\Sigma}_{1} \tilde{V}_{1}^{*}-U_{1} \Sigma_{1} V_{1}^{*} & =\tilde{U}_{1} \tilde{\Sigma}_{1} \tilde{V}_{1}^{*}\left(I_{n}-D_{2}^{-1}\right)+\left(D_{1}^{*}-I_{m}\right) U_{1} \Sigma_{1} V_{1}^{*} \\
& =\left(I_{m}-D_{1}^{-*}\right) \tilde{U}_{1} \tilde{\Sigma}_{1} \tilde{V}_{1}^{*}+U_{1} \Sigma_{1} V_{1}^{*}\left(D_{2}-I_{n}\right) .
\end{aligned}
$$

By (4.3) we obtain

$$
\begin{gathered}
\tilde{\Sigma}_{1} \tilde{V}_{1}^{*} N^{-1} V_{1}-\tilde{U}_{1}^{*} M U_{1} \Sigma_{1}=\tilde{\Sigma}_{1} \tilde{V}_{1}^{*}\left(I_{n}-D_{2}^{-1}\right) N^{-1} V_{1}+\tilde{U}_{1}^{*} M\left(D_{1}^{*}-I_{m}\right) U_{1} \Sigma_{1} \\
U_{1}^{*} M \tilde{U}_{1} \tilde{\Sigma}_{1}-\Sigma_{1} V_{1}^{*} N^{-1} \tilde{V}_{1}=U_{1}^{*} M\left(I_{m}-D_{1}^{-*}\right) \tilde{U}_{1} \tilde{\Sigma}_{1}+\Sigma_{1} V_{1}^{*}\left(D_{2}-I_{n}\right) N^{-1} \tilde{V}_{1} \\
U_{2}^{*} M \tilde{U}_{1} \tilde{\Sigma}_{1}=U_{2}^{*} M\left(I_{m}-D_{1}^{-*}\right) \tilde{U}_{1} \tilde{\Sigma}_{1} \\
\tilde{U}_{2}^{*} M U_{1} \Sigma_{1}=-\tilde{U}_{2}^{*} M\left(D_{1}^{*}-I_{m}\right) U_{1} \Sigma_{1} \\
\tilde{\Sigma}_{1} \tilde{V}_{1}^{*} N^{-1} V_{2}=\tilde{\Sigma}_{1} \tilde{V}_{1}^{*}\left(I_{n}-D_{2}^{-1}\right) N^{-1} V_{2} \\
\Sigma_{1} V_{1}^{*} N^{-1} \tilde{V}_{2}=-\Sigma_{1} V_{1}^{*}\left(D_{2}-I_{n}\right) N^{-1} \tilde{V}_{2}
\end{gathered}
$$

from which one may deduce that

$$
\tilde{V}_{1}^{*} N^{-1} V_{1} \Sigma_{1}^{-1}-\tilde{\Sigma}_{1}^{-1} \tilde{U}_{1}^{*} M U_{1}
$$




$$
=\tilde{V}_{1}^{*}\left(I_{n}-D_{2}^{-1}\right) N^{-1} V_{1} \Sigma_{1}^{-1}+\tilde{\Sigma}_{1}^{-1} \tilde{U}_{1}^{*} M\left(D_{1}^{*}-I_{m}\right) U_{1},
$$

$$
\begin{gathered}
\tilde{\Sigma}_{1}^{-1} \tilde{U}_{1}^{*} M U_{2}=\tilde{\Sigma}_{1}^{-1} \tilde{U}_{1}^{*}\left(I_{m}-D_{1}^{-1}\right) M U_{2} \\
-\tilde{V}_{2}^{*} N^{-1} V_{1} \Sigma_{1}^{-1}=\tilde{V}_{2}^{*} N^{-1}\left(D_{2}^{*}-I_{n}\right) V_{1} \Sigma_{1}^{-1}
\end{gathered}
$$

$\Sigma_{1}^{-1} U_{1}^{*} M \tilde{U}_{1}-V_{1}^{*} N^{-1} \tilde{V}_{1} \tilde{\Sigma}_{1}^{-1}$

$$
=\Sigma_{1}^{-1} U_{1}^{*} M\left(I_{m}-D_{1}^{-*}\right) \tilde{U}_{1}+V_{1}^{*}\left(D_{2}-I_{n}\right) N^{-1} \tilde{V}_{1} \tilde{\Sigma}_{1}^{-1}
$$

$$
-\Sigma_{1}^{-1} U_{1}^{*} M \tilde{U}_{2}=\Sigma_{1}^{-1} U_{1}^{*}\left(D_{1}-I_{m}\right) M \tilde{U}_{2},
$$

$$
V_{2}^{*} N^{-1} \tilde{V}_{1} \tilde{\Sigma}_{1}^{-1}=V_{2}^{*} N^{-1}\left(I_{n}-D_{2}^{-*}\right) \tilde{V}_{1} \tilde{\Sigma}_{1}^{-1} .
$$

By (3.7) and (4.4)-(4.6) we obtain

$$
\begin{aligned}
\| B_{M N}^{\dagger} & -A_{M N}^{\dagger} \|_{(N M)} \\
& =\left\|\left(\begin{array}{cc}
\mathcal{L} & \tilde{\Sigma}_{1}^{-1} \tilde{U}_{1}^{*}\left(I_{m}-D_{1}^{-1}\right) M U_{2} \\
\tilde{V}_{2}^{*} N^{-1}\left(D_{2}^{*}-I_{n}\right) V_{1} \Sigma_{1}^{-1} & \mathbf{0}
\end{array}\right)\right\| \\
& \leq\left\|\left(\begin{array}{cc}
-\tilde{\Sigma}_{1}^{-1} \tilde{U}_{1}^{*} M\left(D_{1}^{*}-I_{m}\right) U_{1} & \tilde{\Sigma}_{1}^{-1} \tilde{U}_{1}^{*}\left(I_{m}-D_{1}^{-1}\right) M U_{2} \\
\mathbf{0} & \mathbf{0}
\end{array}\right)\right\| \\
& +\left\|\left(\begin{array}{cc}
-\tilde{V}_{1}^{*}\left(I_{n}-D_{2}^{-1}\right) N^{-1} V_{1} \Sigma_{1}^{-1} & \mathbf{0} \\
\tilde{V}_{2}^{*} N^{-1}\left(D_{2}^{*}-I_{n}\right) V_{1} \Sigma_{1}^{-1} & \mathbf{0}
\end{array}\right)\right\| \\
& \leq\left\|\left(\begin{array}{cc}
\mathcal{M} & \tilde{\Sigma}_{1}^{-1} \tilde{U}_{1}^{*} M^{\frac{1}{2}} M^{-\frac{1}{2}}\left(I_{m}-D_{1}^{-1}\right) M^{\frac{1}{2}} M^{\frac{1}{2}} U_{2} \\
\mathbf{0} & \mathbf{0}
\end{array}\right)\right\| \\
& +\left\|\left(\begin{array}{cc}
-\tilde{V}_{1}^{*} N^{-\frac{1}{2}} N^{\frac{1}{2}}\left(I_{n}-D_{2}^{-1}\right) N^{-\frac{1}{2}} N^{-\frac{1}{2}} V_{1} \Sigma_{1}^{-1} & \mathbf{0} \\
\tilde{V}_{2}^{*} N^{-\frac{1}{2}} N^{-\frac{1}{2}}\left(D_{2}^{*}-I_{n}\right) N^{\frac{1}{2}} N^{-\frac{1}{2}} V_{1} \Sigma_{1}^{-1} & \mathbf{0}
\end{array}\right)\right\|,
\end{aligned}
$$

where

$$
\begin{gathered}
\mathcal{L} \equiv-\tilde{V}_{1}^{*}\left(I_{n}-D_{2}^{-1}\right) N^{-1} V_{1} \Sigma_{1}^{-1}-\tilde{\Sigma}_{1}^{-1} \tilde{U}_{1}^{*} M\left(D_{1}^{*}-I_{m}\right) U_{1}, \\
\mathcal{M} \equiv-\tilde{\Sigma}_{1}^{-1} \tilde{U}_{1}^{*} M^{\frac{1}{2}} M^{\frac{1}{2}}\left(D_{1}^{*}-I_{m}\right) M^{-\frac{1}{2}} M^{\frac{1}{2}} U_{1} .
\end{gathered}
$$

This implies (4.1) holds.

Remark 4.2. If we take $M=N=I$, then Theorem 4.1 reduces to

$$
\left\|B^{\dagger}-A^{\dagger}\right\| \leq \max \left\{\left\|A^{\dagger}\right\|_{2},\left\|B^{\dagger}\right\|_{2}\right\} \Psi\left(D_{1}, D_{2}\right),
$$


where

$$
\Psi\left(D_{1}, D_{2}\right)=\left\|I_{m}-D_{1}\right\|+\left\|I_{m}-D_{1}^{-1}\right\|+\left\|I_{n}-D_{2}\right\|+\left\|I_{n}-D_{2}^{-1}\right\|,
$$

which is the result of Theorem 4.1 in [3].

For the $Q_{(N M)}$-norm we obtain the following bound:

Theorem 4.3. Let $A \in \mathbb{C}^{m \times n}$ and $B=D_{1}^{*} A D_{2}$, where $D_{1}$ and $D_{2}$ are $m \times m$ and $n \times n$ nonsingular matrices, respectively. Then

$$
\left\|B_{M N}^{\dagger}-A_{M N}^{\dagger}\right\|_{Q(N M)} \leq \sqrt{\frac{3}{2}} \max \left\{\left\|A_{M N}^{\dagger}\right\|_{N M},\left\|B_{M N}^{\dagger}\right\|_{N M}\right\} \Phi_{1}\left(D_{1}, D_{2}\right),
$$

where

$$
\begin{aligned}
\Phi_{1}\left(D_{1}, D_{2}\right)= & {\left[\left\|I_{m}-D_{1}^{*}\right\|_{Q(M M)}^{2}+\left\|I_{m}-D_{1}^{-*}\right\|_{Q(M M)}^{2}\right.} \\
& \left.+\left\|I_{n}-D_{2}\right\|_{Q(N N)}^{2}+\left\|I_{n}-D_{2}^{-1}\right\|_{Q(N N)}^{2}\right]^{\frac{1}{2}}
\end{aligned}
$$

Proof. By (3.8), (4.4)-(4.9) and Lemma 2.1 we can derive

$2\left\|B_{M N}^{\dagger}-A_{M N}^{\dagger}\right\|_{Q(N M)}^{2}$

$$
\begin{aligned}
& \leq\left\|\tilde{V}_{1}^{*}\left(I_{n}-D_{2}^{-1}\right) N^{-1} V_{1} \Sigma_{1}^{-1}+\tilde{\Sigma}_{1}^{-1} \tilde{U}_{1}^{*} M\left(D_{1}^{*}-I_{m}\right) U_{1}\right\|_{Q}^{2} \\
& +\left\|\tilde{\Sigma}_{1}^{-1} \tilde{U}_{1}^{*}\left(I_{m}-D_{1}^{-1}\right) M U_{2}\right\|_{Q}^{2}+\left\|\tilde{V}_{2}^{*} N^{-1}\left(D_{2}^{*}-I_{n}\right) V_{1} \Sigma_{1}^{-1}\right\|_{Q}^{2} \\
& +\left\|\Sigma_{1}^{-1} U_{1}^{*} M\left(I_{m}-D_{1}^{-*}\right) \tilde{U}_{1}+V_{1}^{*}\left(D_{2}-I_{n}\right) N^{-1} \tilde{V}_{1} \tilde{\Sigma}_{1}^{-1}\right\|_{Q}^{2} \\
& +\left\|\Sigma_{1}^{-1} U_{1}^{*}\left(D_{1}-I_{m}\right) M \tilde{U}_{2}\right\|_{Q}^{2}+\left\|V_{2}^{*} N^{-1}\left(I_{n}-D_{2}^{-*}\right) \tilde{V}_{1} \tilde{\Sigma}_{1}^{-1}\right\|_{Q}^{2} \\
& \leq\left(\frac{1}{\sigma_{r}}\left\|\tilde{V}_{1}^{*}\left(I_{n}-D_{2}^{-1}\right) N^{-1} V_{1}\right\|_{Q}+\frac{1}{\tilde{\sigma}_{r}}\left\|\tilde{U}_{1}^{*} M\left(D_{1}^{*}-I_{m}\right) U_{1}\right\|_{Q}\right)^{2} \\
& +\left(\frac{1}{\sigma_{r}}\left\|U_{1}^{*} M\left(I_{m}-D_{1}^{-*}\right) \tilde{U}_{1}\right\|_{Q}+\frac{1}{\tilde{\sigma}_{r}}\left\|V_{1}^{*}\left(D_{2}-I_{n}\right) N^{-1} \tilde{V}_{1}\right\|_{Q}\right)^{2} \\
& +\frac{1}{\tilde{\sigma}_{r}^{2}}\left(\left\|\tilde{U}_{1}^{*}\left(I_{m}-D_{1}^{-1}\right) M U_{2}\right\|_{Q}^{2}+\left\|V_{2}^{*} N^{-1}\left(I_{n}-D_{2}^{-*}\right) \tilde{V}_{1}\right\|_{Q}^{2}\right) \\
& +\frac{1}{\sigma_{r}^{2}}\left(\left\|\tilde{V}_{2}^{*} N^{-1}\left(D_{2}^{*}-I_{n}\right) V_{1}\right\|_{Q}^{2}+\left\|U_{1}^{*}\left(D_{1}-I_{m}\right) M \tilde{U}_{2}\right\|_{Q}^{2}\right) \\
& \leq\left(\frac{2}{\sigma_{r}^{2}}\left\|\tilde{V}_{1}^{*}\left(I_{n}-D_{2}^{-1}\right) N^{-1} V_{1}\right\|_{Q}^{2}+\frac{2}{\tilde{\sigma}_{r}^{2}}\left\|\tilde{U}_{1}^{*} M\left(D_{1}^{*}-I_{m}\right) U_{1}\right\|_{Q}^{2}\right) \\
& +\left(\frac{2}{\sigma_{r}^{2}}\left\|U_{1}^{*} M\left(I_{m}-D_{1}^{-*}\right) \tilde{U}_{1}\right\|_{Q}^{2}+\frac{2}{\tilde{\sigma}_{r}^{2}}\left\|V_{1}^{*}\left(D_{2}-I_{n}\right) N^{-1} \tilde{V}_{1}\right\|_{Q}^{2}\right) \\
& +\frac{1}{\tilde{\sigma}_{r}^{2}}\left(\left\|\tilde{U}_{1}^{*}\left(I_{m}-D_{1}^{-1}\right) M U_{2}\right\|_{Q}^{2}+\left\|V_{2}^{*} N^{-1}\left(I_{n}-D_{2}^{-*}\right) \tilde{V}_{1}\right\|_{Q}^{2}\right) \\
& +\frac{1}{\sigma_{r}^{2}}\left(\left\|\tilde{V}_{2}^{*} N^{-1}\left(D_{2}^{*}-I_{n}\right) V_{1}\right\|_{Q}^{2}+\left\|U_{1}^{*}\left(D_{1}-I_{m}\right) M \tilde{U}_{2}\right\|_{Q}^{2}\right)
\end{aligned}
$$




$$
\begin{aligned}
\leq & \max \left\{\frac{1}{\sigma_{r}^{2}}, \frac{1}{\tilde{\sigma}_{r}^{2}}\right\}\left[\left(2\left\|\tilde{V}_{1}^{*}\left(I_{n}-D_{2}^{-1}\right) N^{-1} V_{1}\right\|_{Q}^{2}\right.\right. \\
+ & \left.\left\|V_{2}^{*} N^{-1}\left(I_{n}-D_{2}^{-*}\right) \tilde{V}_{1}\right\|_{Q}^{2}\right)+\left(2 \| \tilde{U}_{1}^{*} M\left(D_{1}^{*}\right.\right. \\
& \left.\left.-I_{m}\right) U_{1}\left\|_{Q}^{2}+\right\| U_{1}^{*}\left(D_{1}^{*}-I_{m}\right) M \tilde{U}_{2} \|_{Q}^{2}\right)+\left(2\left\|U_{1}^{*} M\left(I_{m}-D_{1}^{-*}\right) \tilde{U}_{1}\right\|_{Q}^{2}\right. \\
+ & \left.\left\|\tilde{U}_{1}^{*}\left(I_{m}-D_{1}^{-1}\right) M U_{2}\right\|_{Q}^{2}\right)+\left(2\left\|V_{1}^{*}\left(D_{2}-I_{n}\right) N^{-1} \tilde{V}_{1}\right\|_{Q}^{2}\right. \\
+ & \left.\left.\frac{1}{\sigma_{r}^{2}}\left(\left\|\tilde{V}_{2}^{*} N^{-1}\left(D_{2}^{*}-I_{n}\right) V_{1}\right\|_{Q}^{2}\right)\right)\right] \\
\leq & 3 \max \left\{\frac{1}{\sigma_{r}^{2}}, \frac{1}{\tilde{\sigma}_{r}^{2}}\right\}\left[\left\|I_{m}-D_{1}^{*}\right\|_{Q(M M)}^{2}+\left\|I_{m}-D_{1}^{-*}\right\|_{Q(M M)}^{2}+\left\|I_{n}-D_{2}\right\|_{Q(N N)}^{2}\right. \\
+ & \left.\left\|I_{n}-D_{2}^{-1}\right\|_{Q(N N)}^{2}\right] \\
= & 3 \max \left\{\left\|A_{M N}^{\dagger}\right\|_{N M}^{2},\left\|B_{M N}^{\dagger}\right\|_{N M}^{2}\right\} \Phi\left(D_{1}, D_{2}\right)^{2},
\end{aligned}
$$

where $\Phi_{1}\left(D_{1}, D_{2}\right)$ equals

$$
\left[\left\|I_{m}-D_{1}^{*}\right\|_{Q(M M)}^{2}+\left\|I_{m}-D_{1}^{-*}\right\|_{Q(M M)}^{2}+\left\|I_{n}-D_{2}\right\|_{Q(N N)}^{2}+\left\|I_{n}-D_{2}^{-1}\right\|_{Q(N N)}^{2}\right]^{\frac{1}{2}} .
$$

Hence,

$$
\left\|B_{M N}^{\dagger}-A_{M N}^{\dagger}\right\|_{Q(N M)} \leq \sqrt{\frac{3}{2}} \max \left\{\left\|A_{M N}^{\dagger}\right\|_{N M},\left\|B_{M N}^{\dagger}\right\|_{N M}\right\} \Phi_{1}\left(D_{1}, D_{2}\right),
$$

which derives the desired result. $\square$

Remark 4.4. If we take $M=N=I$, then Theorem 4.3 reduces to

$$
\left\|B^{\dagger}-A^{\dagger}\right\|_{Q} \leq \sqrt{\frac{3}{2}} \max \left\{\left\|A^{\dagger}\right\|_{2},\left\|B^{\dagger}\right\|_{2}\right\} \Phi\left(D_{1}, D_{2}\right),
$$

where

$$
\Phi\left(D_{1}, D_{2}\right)=\left[\left\|I_{m}-D_{1}\right\|_{Q}^{2}+\left\|I_{m}-D_{1}^{-1}\right\|_{Q}^{2}+\left\|I_{n}-D_{2}\right\|_{Q}^{2}+\left\|I_{n}-D_{2}^{-1}\right\|_{Q}^{2}\right]^{\frac{1}{2}},
$$

which is the result of Theorem 4.2 in [3].

For the $F_{(N M)}$-norm we obtain the following bound:

Theorem 4.5. Let $A \in \mathbb{C}^{m \times n}$ and $B=D_{1}^{*} A D_{2}$, where $D_{1}$ and $D_{2}$ are respectively $m \times m$ and $n \times n$ nonsingular matrices. Then

(4.13) $\left\|B_{M N}^{\dagger}-A_{M N}^{\dagger}\right\|_{F(N M)} \leq \max \left\{\left\|A_{M N}^{\dagger}\right\|_{N M},\left\|B_{M N}^{\dagger}\right\|_{N M}\right\} \varphi_{1}\left(D_{1}, D_{2}\right)$,

where $\varphi_{1}\left(D_{1}, D_{2}\right)$ equals $\left\|I_{m}-D_{1}^{*}\right\|_{F(M M)}^{2}+\left\|I_{m}-D_{1}^{-*}\right\|_{F(M M)}^{2}+\left\|I_{n}-D_{2}\right\|_{F(N N)}^{2}+$ $\left.\left\|I_{n}-D_{2}^{-1}\right\|_{F(N N)}^{2}\right]^{\frac{1}{2}}$. 
Proof. It follows from (4.12) and Lemma 1.2 of [5] that

$$
\begin{aligned}
& 2\left\|B_{M N}^{\dagger}-A_{M N}^{\dagger}\right\|_{F(N M)}^{2} \\
& \leq \max \left\{\frac{1}{\sigma_{r}^{2}}, \frac{1}{\tilde{\sigma}_{r}^{2}}\right\}\left[\left(2\left\|\tilde{V}_{1}^{*}\left(I_{n}-D_{2}^{-1}\right) N^{-1} V_{1}\right\|_{F}^{2}+\left\|V_{2}^{*} N^{-1}\left(I_{n}-D_{2}^{-*}\right) \tilde{V}_{1}\right\|_{F}^{2}\right)\right. \\
& +\left(2\left\|\tilde{U}_{1}^{*} M\left(D_{1}^{*}-I_{m}\right) U_{1}\right\|_{F}^{2}+\left\|U_{1}^{*}\left(D_{1}-I_{m}\right) M \tilde{U}_{2}\right\|_{F}^{2}\right) \\
& +\left(2\left\|U_{1}^{*} M\left(I_{m}-D_{1}^{-*}\right) \tilde{U}_{1}\right\|_{F}^{2}+\left\|\tilde{U}_{1}^{*}\left(I_{m}-D_{1}^{-1}\right) M U_{2}\right\|_{F}^{2}\right) \\
& \left.+\left(2\left\|V_{1}^{*}\left(D_{2}-I_{n}\right) N^{-1} \tilde{V}_{1}\right\|_{F}^{2}+\frac{1}{\sigma_{r}^{2}}\left(\left\|\tilde{V}_{2}^{*} N^{-1}\left(D_{2}^{*}-I_{n}\right) V_{1}\right\|_{F}^{2}\right)\right)\right] \\
& \leq 2 \max \left\{\frac{1}{\sigma_{r}^{2}}, \frac{1}{\tilde{\sigma}_{r}^{2}}\right\}\left(\left\|I_{m}-D_{1}^{*}\right\|_{F(M M)}^{2}+\left\|I_{m}-D_{1}^{-*}\right\|_{F(M M)}^{2}+\left\|I_{n}-D_{2}\right\|_{F(N N)}^{2}\right) \\
& \left.+\left\|I_{n}-D_{2}^{-1}\right\|_{F(N N)}^{2}\right) \\
& =2 \max \left\{\left\|A_{M N}^{\dagger}\right\|_{N M}^{2},\left\|B_{M N}^{\dagger}\right\|_{N M}^{2}\right\} \varphi_{1}\left(D_{1}, D_{2}\right)^{2}
\end{aligned}
$$

which implies that (4.13) holds. $\square$

REMARK 4.6. If we take $M=N=I$, then Theorem 4.5 reduces to

$$
\left\|B^{\dagger}-A^{\dagger}\right\|_{F} \leq \max \left\{\left\|A^{\dagger}\right\|_{2},\left\|B^{\dagger}\right\|_{2}\right\} \varphi\left(D_{1}, D_{2}\right),
$$

where

$$
\varphi\left(D_{1}, D_{2}\right)=\left[\left\|I_{m}-D_{1}\right\|_{F}^{2}+\left\|I_{m}-D_{1}^{-1}\right\|_{F}^{2}+\left\|I_{n}-D_{2}\right\|_{F}^{2}+\left\|I_{n}-D_{2}^{-1}\right\|_{F}^{2}\right]^{\frac{1}{2}},
$$

which is the result of Theorem 3.1 in [5].

REMARK 4.7. We note that a multiplicative perturbation can also be viewed as an additive one. The example in Remark 4.1 of [3] and Example 3 in [5] have shown that the multiplicative bounds (4.11), (4.13) are better than the additive bounds (3.29) and (3.26), respectively.

5. Numerical examples. In this section, we give some simple numerical examples to illustrate the optimality of our given bounds under the weighted $Q$ - norm and $F$ - norm, respectively. Examples 1 and 2 in [5] show the optimality of the additive perturbation bounds in (3.15) and (3.26), respectively. The example in Remark 3.3 of [3] shows the approximate optimality of the perturbation bound in (3.14). The following examples will illustrate that the bounds in (3.29), (4.11) and (4.13) are approximately optimal.

ExAmPLE 5.1. Let

$$
A=\left(\begin{array}{lll}
1 & 0 & 0 \\
0 & 1 & 0 \\
0 & 0 & 2
\end{array}\right), \quad B=\left(\begin{array}{ccc}
1.000001 & 0 & 0 \\
0 & 1.000001 & 0 \\
0 & 0 & 2.000001
\end{array}\right)
$$




$$
M=\left(\begin{array}{lll}
1 & 0 & 0 \\
0 & 1 & 0 \\
0 & 0 & 4
\end{array}\right), \quad N=\left(\begin{array}{lll}
1 & 0 & 0 \\
0 & 1 & 0 \\
0 & 0 & 4
\end{array}\right) .
$$

We take the Ky-Fan 2-2 norm as a $Q$-norm (for details about the Ky-Fan norm please see [3]). Then

$$
\begin{gathered}
\left\|B_{M N}^{\dagger}-A_{M N}^{\dagger}\right\|_{Q(N M)}=\frac{\sqrt{2}}{1000001}, \\
\sqrt{3}\left\|A_{M N}^{\dagger}\right\|_{N M}\left\|B_{M N}^{\dagger}\right\|_{N M}\|E\|_{Q(M N)} \doteq \frac{\sqrt{2}}{1000001},
\end{gathered}
$$

which implies the bound in (3.29) is approximate to the optimal bound.

EXAMPLE 5.2. Let

$$
\begin{gathered}
A=\left(\begin{array}{ccc}
10^{7} & 0 & 0 \\
0 & 10^{7} & 0 \\
0 & 0 & 2 \cdot 10^{7}
\end{array}\right), \quad D_{1}=I, D_{2}=\left(\begin{array}{ccc}
\frac{1}{2} & 0 & 0 \\
0 & 2 & 0 \\
0 & 0 & \frac{1}{2}
\end{array}\right), \\
M=\left(\begin{array}{lll}
1 & 0 & 0 \\
0 & 1 & 0 \\
0 & 0 & 3
\end{array}\right), N=\left(\begin{array}{lll}
1 & 0 & 0 \\
0 & 1 & 0 \\
0 & 0 & 3
\end{array}\right) .
\end{gathered}
$$

We take the Ky-Fan 2-2 norm as a $Q$-norm. Then

$$
\begin{gathered}
\left\|B_{M N}^{\dagger}-A_{M N}^{\dagger}\right\|_{Q(N M)}=\sqrt{2} \times 10^{-7}, \\
\sqrt{\frac{3}{2}} \max \left\{\left\|A_{M N}^{\dagger}\right\|_{N M},\left\|B_{M N}^{\dagger}\right\|_{N M}\right\} \Phi_{1}\left(D_{1}, D_{2}\right) \doteq \sqrt{2} \times 10^{-7},
\end{gathered}
$$

which implies the bound (4.11) is approximate to the optimal bound.

EXAMPLE 5.3. Let

$$
A=\left(\begin{array}{cc}
10^{9} & 0 \\
0 & 10^{9}
\end{array}\right), D_{1}=I, D_{2}=\left(\begin{array}{cc}
1 & 0 \\
0 & \frac{1}{2}
\end{array}\right), M=\left(\begin{array}{ll}
1 & 0 \\
0 & 2
\end{array}\right), N=\left(\begin{array}{ll}
1 & 0 \\
0 & 2
\end{array}\right) .
$$

Then

$$
\begin{gathered}
\left\|B_{M N}^{\dagger}-A_{M N}^{\dagger}\right\|_{F(N M)}=1 \times 10^{-9} \\
\max \left\{\left\|A_{M N}^{\dagger}\right\|_{N M},\left\|B_{M N}^{\dagger}\right\|_{N M}\right\} \varphi_{1}\left(D_{1}, D_{2}\right) \doteq 1 \times 10^{-9},
\end{gathered}
$$

which implies the bound (4.13) is approximate to the optimal bound. 
6. Concluding remarks. Problems about the weighted Moore-Penrose inverse arise in many fields, e.g., weighted generalized inverse problems and the weighted least squares problem, etc. Of them, an interesting one is that when the original matrix $A$ is perturbed to be $B=A+E$, how does the weighted Moore-Penrose inverse transform? In this paper we have considered this problem and by using the $(M, N)$ weighted singular value decomposition $(M N-S V D)$ some optimal bounds for additive and multiplicative perturbation under the norms $\|\cdot\|_{(M N)},\|\cdot\|_{F(M N)}$ and $\|\cdot\|_{Q(M N)}$ are presented, respectively. Our results extend the corresponding ones in [3] and [5].

Acknowledgment. The authors would like to thank the referee for his/her kind suggestions.

\section{REFERENCES}

[1] R.B. Bapat, S.K. Jain, and S. Pati. Weighted Moore-Penrose inverse of a Boolean matrix. Linear Algebra Appl., 255:267-279, 1997.

[2] A. Ben-Israel and T.N.E. Greville. Generalized Inverses: Theory and Applications, 2 nd edition. Springer, New York, 2003.

[3] L.X. Cai, W.W. Xu, and W. Li. Additive and multiplicative perturbation bounds for the MoorePenrose inverse. Linear Algebra Appl., 434:480-489, 2011.

[4] V. Mehrmann. A symplectic orthogonal method for single input or single output discrete time optimal linear quadratic control problems. SIAM J. Matrix Anal. Appl., 9:221-247, 1988.

[5] L.S. Meng and B. Zheng. The optimal perturbation bounds of the Moore-Penrose inverse under the Frobenius norm. Linear Algebra Appl., 432:956-963, 2010.

[6] M.D. Petković and P.S. Stanimirović. Symbolic computation of the Moore-Penrose inverse using a partitioning method. Int. J. Comput. Math., 82:355-367, 2005.

[7] M.D. Petković, P.S. Stanimirović, and M.B. Tasić. Effective partitioning method for computing weighted Moore-Penrose inverse. Comput. Math. Appl., 55:1720-1734, 2008.

[8] C.R. Rao and S.K. Mitra. Generalized Inverses of Matrices and its Applications. Wiley, New York, 1971.

[9] C.R. Rao and M.B. Rao. Matrix Algebra and its Applications to Statistics and Econometrics. World Scientific, Hong Kong, 1998.

[10] P.S. Stanimirović and M.B. Tasić. Partitioning method for rational and polynomial matrices. Appl. Math. Comput., 155:137-163, 2004.

[11] M.B. Tasić, P.S. Stanimirović, and M.D. Petković. Symbolic computation of weighted MoorePenrose inverse using partitioning method. Appl. Math. Comput., 189:615-640, 2007.

[12] G.R. Wang, Y.M. Wei, and S.Z. Qiao, Generalized Inverses: Theory and Computations. Science, Beijing, 2004.

[13] P.A. Wedin. Perturbation theory for pseudo-inverses. Nordisk Tidskr. Informationsbehandling (BIT), 13:217-232, 1973.

[14] Y.M. Wei and H.B. Wu. Expression for the perturbation of the weighted Moore-Penrose inverse. Comput. Math. Appl., 39:13-18, 2000.

[15] H. Yang and H.Y. Li. Weighted polar decomposition and WGL partial ordering of rectangular complex matrices. SIAM J. Matrix Anal. Appl., 30:898-924, 2008. 